\title{
ARTICLE
}

\section{Haemodynamically dependent valvulogenesis of zebrafish heart is mediated by flow-dependent expression of $\mathrm{miR}-21$}

Toshihiro Banjo1, Janin Grajcarek ${ }^{1,2}$, Daisuke Yoshino ${ }^{3}$, Hideto Osada', Kota Y. Miyasaka1,4, Yasuyuki S. Kida ${ }^{5}$, Yosuke Ueki ${ }^{6}$, Kazuaki Nagayama ${ }^{7}$, Koichi Kawakami ${ }^{8}$, Takeo Matsumoto ${ }^{7}$, Masaaki Sato ${ }^{6}$ \& Toshihiko Ogura ${ }^{1,4}$

Heartbeat is required for normal development of the heart, and perturbation of intracardiac flow leads to morphological defects resembling congenital heart diseases. These observations implicate intracardiac haemodynamics in cardiogenesis, but the signalling cascades connecting physical forces, gene expression and morphogenesis are largely unknown. Here we use a zebrafish model to show that the microRNA, miR-21, is crucial for regulation of heart valve formation. Expression of miR-21 is rapidly switched on and off by blood flow. Vasoconstriction and increasing shear stress induce ectopic expression of miR-21 in the head vasculature and heart. Flow-dependent expression of mir-21 governs valvulogenesis by regulating the expression of the same targets as mouse/human miR-21 (sprouty, pdcd4, ptenb) and induces cell proliferation in the valve-forming endocardium at constrictions in the heart tube where shear stress is highest. We conclude that miR-21 is a central component of a flow-controlled mechanotransduction system in a physicogenetic regulatory loop.

\footnotetext{
${ }^{1}$ Graduate School of Life Sciences, Tohoku University, 2-1-1 Katahira, Aoba, Sendai, Miyagi 980-8577, Japan. ${ }^{2}$ Faculty of Biosciences, Universität Heidelberg, Im Neuenheimer Feld 234, D-69120 Heidelberg, Germany. ${ }^{3}$ Biological Nanoscale Reactive Flow Laboratory, Institute of Fluid Science, Tohoku University, 2-11, Katahira, Aoba, Sendai, Miyagi 980-8577, Japan. ${ }^{4}$ Department of Developmental Neurobiology, Institute of Development, Aging and Cancer, Tohoku University, 4-1 Seiryo, Aoba, Sendai, Miyagi 980-8575, Japan. ${ }^{5}$ Mesenchymal Stem Cell Dynamics Research Team, Research Center for Stem Cell Engineering, National Institutes of Advanced Industrial Science and Technology, 1-1-1, Higashi, Tsukuba, Ibaraki 305-8562, Japan. ${ }^{6}$ Department of Biomedical Engineering, Graduate School of Biomedical Engineering, Tohoku University, 6-6-1, Aoba, Aramaki, Sendai, Miyagi 980-8579, Japan. ${ }^{7}$ Department of Mechanical Engineering, Nagoya Institute of Technology, Gokisho, Showa, Nagoya, Aichi 466-8555, Japan. ${ }^{8}$ Division of Molecular and Developmental Biology, National Institute of Genetics, 1111 Yata, Mishima, Shizuoka 411-8540, Japan. Correspondence and requests for materials should be addressed to T.O. (email: ogura@idac.tohoku.ac.jp).
} 
F ormation of the three-dimensional structure of the heart begins with fusion of bilateral mesodermal planes that subsequently differentiate into beating cardiomyocytes ${ }^{1,2}$. Initially, the cardiomyocytes contract randomly but they soon become synchronized as the heart tube develops. Subsequently, the contractions are coordinated into rhythmic waves along the heart tube generating unidirectional blood flow. In the early valveless stages, one-way flow is generated by a hydroelastic suction pump ${ }^{3}$. Later, an S-shaped bend forms in the tube and the cardiomyocytes proliferate causing bulges, which develop into two distinct chambers, the atrium and ventricle, with constrictions in the outflow tract (OFT) and atrioventricular (AV) canal. Valves develop at these strictures to ensure unidirectional circulation. Throughout these morphogenetic events, blood flow is maintained. As the valves form at constrictions, the valve-forming endocardial cells are subject to higher sheer forces.

Many studies have suggested that haemodynamic stresses provide essential morphogenetic cues for normal cardiogenesis. Perturbation of blood flow by beads in zebrafish heart causes morphological defects like those observed in congenital heart diseases ${ }^{4,5}$. Auman et al. ${ }^{6}$ reported that the blood flow and contractility regulate the shape of cardiomyocytes and the form of the heart. More recently, reversing blood flow has been shown to regulate valvulogenesis via expression of krüppel-like factor $2 a$ (klf2a) (ref. 7), highlighting a putative link between gene expression and mechanical stimuli.

MicroRNAs (miRNAs) are small non-coding RNAs. Primary transcripts of miRNAs are processed by Drosha and Dicer and the products are incorporated into the RNA-induced silencing complex 8 . Target mRNAs are degraded or repressed at the translational level, bypassing the transcriptional control of mRNA $^{9}$. We speculated that this rapid regulation might be active during cardiogenesis to couple morphogenesis with haemodynamic changes. This idea is further supported by reports that several miRNAs are induced in mouse hearts subjected to pressure overload ${ }^{10}$ and in mechanically stretched smooth muscle ${ }^{11}$. Moreover, recent studies have suggested that miRNAs contribute to haemodynamically dependent cardiogenesis $^{12}$ and angiogenesis ${ }^{13}$. $m i R-21$ is highly expressed in malignant tissues and has several target genes, including PDCD4, PTEN and SPRY2. These genes exhibit a tumour-suppressing effect, classifying it as an oncomiR ${ }^{14}$. Accordingly, deletion of mouse miR-21 suppresses lung tumorigenesis by modulating the Ras/MEK/ERK pathway and apoptosis ${ }^{15}$.

miR-21 is also expressed during cardiac remodelling and appears to be functional in cardiovascular diseases ${ }^{16}$. However, stress-induced cardiac remodelling occurs in the absence of $m i R-21$ in the mouse ${ }^{17}$. In this report, we provide evidence that $m i R-21$ functions differently in zebrafish. By sensing blood flow, it regulates heartbeat-dependent valvulogenesis by repression of the same target genes as in the mouse. We conclude that $m i R-21$ is a crucial component of the programme that regulates valve formation in the zebrafish heart, using haemodynamic stimuli as a physical morphogen.

\section{Results}

Expression patterns of zebrafish $m i R-21$. In zebrafish, valvulogenesis is initiated between 24 and $48 \mathrm{~h}$ post-fertilization (hpf) at the AV canal ${ }^{18}$. Within the canal, the pulsatile flow has critical roles for multiple morphogenetic events, including cell shape changes, protrusion of valve leaflets and expression of klf2a (ref. 7). Expression of $m i R-21$ started at $36 \mathrm{hpf}$ in a small subset of the endocardial cells at the AV constriction (Fig. 1a). At $48 \mathrm{hpf}$, this expression became stronger with an additional endocardial spot in the OFT (Fig. 1b). These two domains became evident at $72 \mathrm{hpf}$. The expression patch in the AV canal developed a hole at the centre corresponding to the lumen, while the OFT expression exhibited a streak parallel to the blood flow (Fig. 1c). miR-21 expression in the AV canal was restricted to the endocardial cells and was not observed in the myocardium (Fig. 1d). As cardiogenesis proceeded, similar expression patterns were observed at $120 \mathrm{hpf}$ (Fig. 1e), and even in the valves of more than 1-year-old adult fishes (Fig. 1f,g). The expression patterns are summarized in Fig. $1 \mathrm{~h}$.

Haemodynamically dependent expression of zebrafish miR-21. The presence of miR-21 expression at constricted sections of the
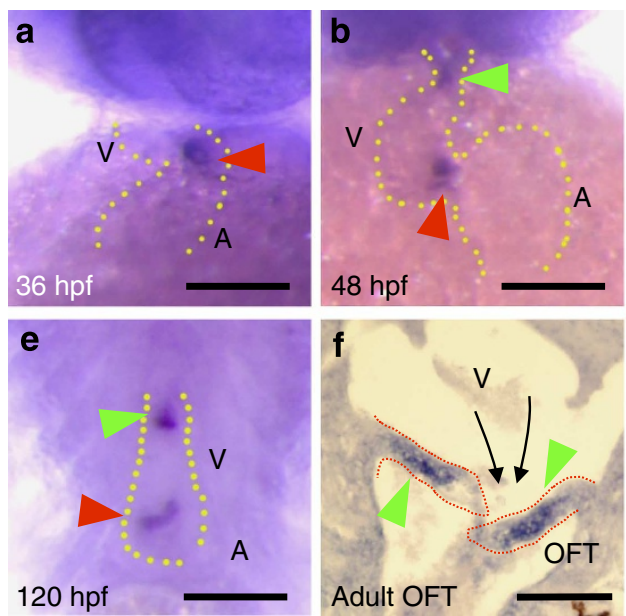
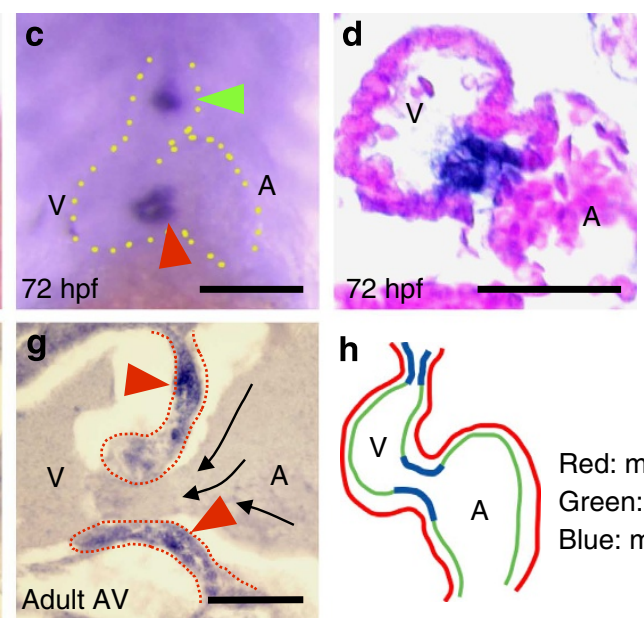

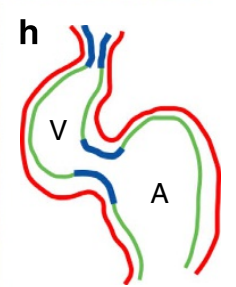

Red: myocardium

Green: endocardium

Blue: miR-21 expression

Figure 1 | Expression patterns of $\mathbf{m i R}-21$. (a) Soon after initiation of blood flow ( $36 \mathrm{hpf}$ ), miR-21 began to be expressed in a subset of the endocardial cells in the AV canal (red arrowhead). A, atrium; V, ventricle. (b) At $48 \mathrm{hpf}$, endocardial expression in the OFT became evident (green arrowhead). Expression at the AV canal was arranged around the canal lumen (red arrowhead). (c,d) The same expression patterns were maintained at $72 \mathrm{hpf}(\mathbf{c})$ and $120 \mathrm{hpf}(\mathbf{e})$. (d) In sections, miR-21 expression was detected only in the endocardial cells, not in the myocardial layer. (f,g) Expression of miR-21 was detected in the valves of adult fish at the OFT (green arrowheads in $\mathbf{f}$ ) and the AV canal (red arrowheads in $\mathbf{g}$ ). Dotted red lines indicate contours of the valves. Black arrows indicate the direction of blood flow. (h) A schematic representation of miR-21 expression only in specific regions of the endocardium of the OFT and the AV canal (blue lines). Scale bars, $50 \mu \mathrm{m}$. 

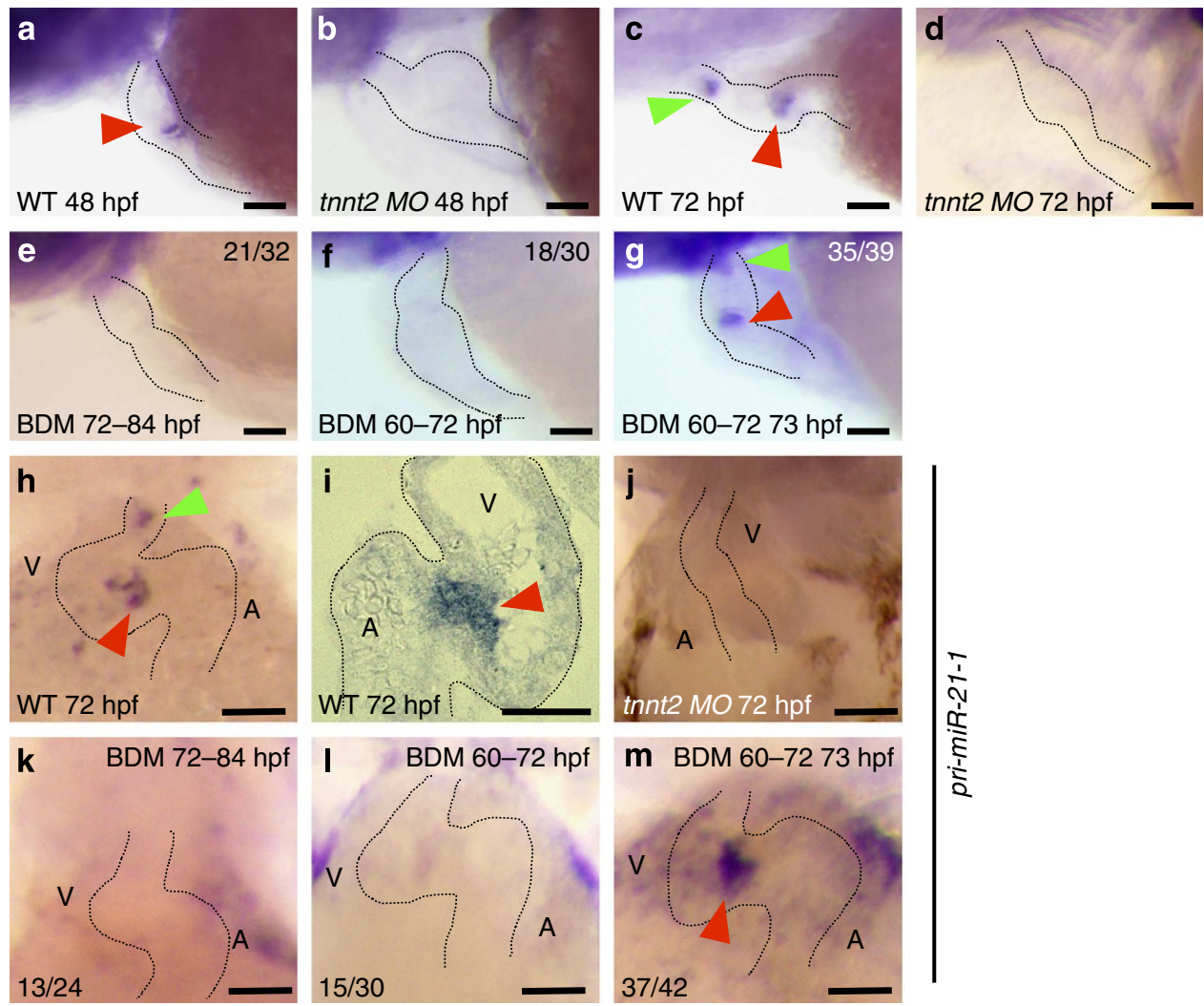

Figure 2 | Effect of heartbeat on expression of $\mathbf{m i R - 2 1}$ in the developing heart tube. (a,c) Normal expression of miR-21 at the OFT (green arrowhead) and the AV canal (red arrowheads) at 48 (a) and $72 \mathrm{hpf}$ (c). Dotted lines outline the heart. (b,d). In the tnnt2 morphants, expression of miR-21 was detected neither at 48 (b) nor at $72 \mathrm{hpf}(\mathbf{d})$. (e-g) Twelve hours arrest of heartbeat (from 72 to $84 \mathrm{hpf}$ in $\mathbf{e}$, and from 60 to $72 \mathrm{hpf}$ in $\mathbf{f}$ ) obliterated the two patches of miR-21 expression, whereas just $1 \mathrm{~h}$ of heartbeat restored the full expression $(\mathbf{g})$. (h-m) pri-miR-21-1 transcripts were expressed in the same patterns at $72 \mathrm{hpf}$ (whole mount in $\mathbf{h}$, and section in $\mathbf{i}$ ). This expression was lost in the tnnt 2 morphant heart $\mathbf{( j )}$ ). Expression of pri-miR-21 disappeared after $12 \mathrm{~h}$ cardiac arrest (from 72 to $84 \mathrm{hpf}$ in $\mathbf{k}$, and from 60 to $72 \mathrm{hpf}$ in I) and $1 \mathrm{~h}$ of heartbeat restored it (red arrowhead in $\mathbf{m}$ ). WT, wild type; MO, morphant; BDM, treated with BDM. Scale bars, $50 \mu \mathrm{m}$.

endocardium suggested that it might be influenced by the blood flow. To investigate this, heartbeat was stopped by injecting a morpholino antisense oligonucleotide (MO) against troponin T2 (tnnt2) (refs 18,19). In this morphant, expression of miR-21 was not observed at either 48 or $72 \mathrm{hpf}$ (Fig. 2b,d), whereas beating hearts showed distinct two expression spots (Fig. 2a,c).

Silent heart mutants (sih), in which tnnt2 is mutated, exhibit morphological defects in their hearts. To exclude the possibility that the loss of the $m i R-21$ expression in the tnnt 2 morphants was a secondary effect of those abnormalities, we stopped the heartbeat temporarily and reversibly using 2,3-butanedione monoxime (BDM, a myosin ATPase inhibitor) ${ }^{20}$. When the heartbeat was arrested for $12 \mathrm{~h}$, from 72 to $84 \mathrm{hpf}$, expression of $m i R-21$ was suppressed at $84 \mathrm{hpf}$ (in 21 of 32 fish, Fig. 2e). Similarly, when the heartbeat was stopped from 60 to $72 \mathrm{hpf}$, expression was lost at $72 \mathrm{hpf}$ (in 18 of 30 fish, Fig. 2f). These data indicate that arrest of the heartbeat for $12 \mathrm{~h}$ is sufficient to cancel expression of $m i R-21$. Conversely, when the heartbeat was reinitiated by washing out the BDM at $72 \mathrm{hpf}, m i R-21$ expression was reestablished at $73 \mathrm{hpf}$ at both valve-forming regions (in 35 of 39 fish, Fig. 2g). Thus, beating for $1 \mathrm{~h}$ can restore the full expression.

Biogenesis of mature miRNAs is regulated at multiple steps from transcription of primary miRNAs to the RNA-induced silencing complex-mediated targeting to mRNAs ${ }^{21}$. To check a transcriptional control of $m i R-21$, we made a probe that specifically recognizes pri-miR-21-1, one of two primary transcripts of zebrafish $m i R-21$ on two different chromosomal loci. At $72 \mathrm{hpf}$, this probe localized at the same positions (Fig. $2 \mathrm{~h}$ ). In sections, expression of pri-miR-21-1 was observed in the valve-forming endocardial cells (Fig. 2i). It was absent in the tnnt 2 morphants at $72 \mathrm{hpf}$ (Fig. 2j). Similarly, $12 \mathrm{~h}$ of arrest cancelled the expression at 84 and $72 \mathrm{hpf}$ (13 of 24 and 15 of 30 fishes, respectively, Fig. $2 \mathrm{k}, \mathrm{l})$, and $1 \mathrm{~h}$ of heartbeat restored it (37 of 42 fishes, Fig. $2 \mathrm{~m}$ ). These data indicate that transcription of pri-miR-21 is regulated in a heartbeat-dependent manner and that the transcriptional response to heart beating is very fast.

It has been shown that the valve formation is dependent on the retrograde flow, rather than blood viscosity, as verified in gata 1 and gata 2 morphants ${ }^{7}$. The gata 1 morphants lack circulating blood cells ${ }^{22}$, hence have lower blood viscosity, whereas the gata2 morphants have reduced retrograde flow at the $\mathrm{AV} \mathrm{canal}^{7}$, due to abnormal vascular development ${ }^{23}$. Valve formation was selectively impaired in the gata 2 morphants with a loss of klf2a expression. In accordance, expression of $m i R-21$ was lost in the gata 2 morphants, not in the gata1 (see Supplementary Fig. S1), supporting the importance of the reversing flow. In addition, injection of MO against neither $k l f 2 a$ nor $t b \times 2 b$ abrogated the expression of $m i R-21$. This suggests independence of the $m i R-21$ expression from klf2a or the AV boundary formation in the myocardium $^{24}$ (see Supplementary Fig. S1).

In an opposite approach, we bathed embryos in water containing $2 \mathrm{mM}$ epinephrine at $51.5 \mathrm{hpf}$ for $1.5 \mathrm{~h}$ to elevate the heart rate along with vasoconstriction. This stimulation should increase the mechanical stress to the valve-forming endocardial cells and the vascular endothelium in the periphery. After the 

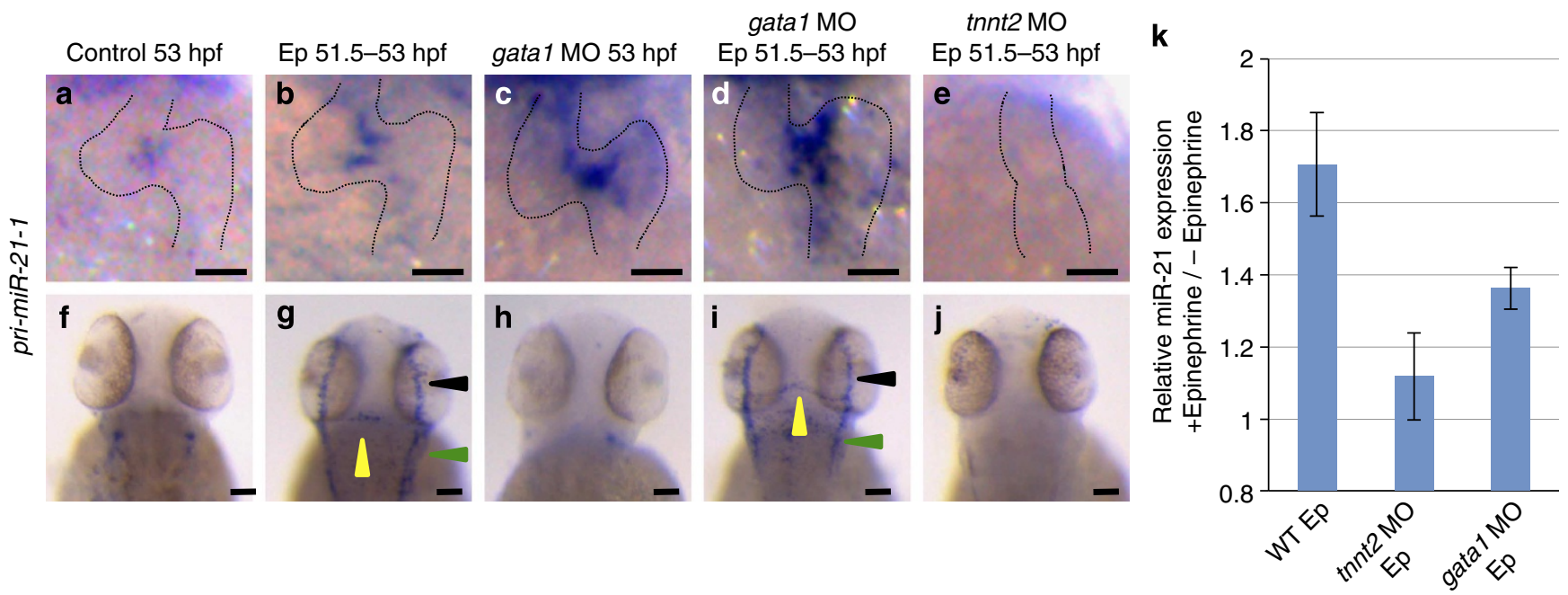

Figure 3 | Expression patterns of pri-miR-21-1, the primary transcript of $\mathbf{m i R}-\mathbf{2 1}$. Expression of pri-miR-21-1 in the heart (a-e) and the head vasculature $(\mathbf{f}-\mathbf{j})$ at $53 \mathrm{hpf}$. The WT (a,b,f and $\mathbf{g})$, gata1 morphants (c,d,h and $\mathbf{i})$ and tnnt2 morphants (e,j) were stimulated by epinephrine (Epi) from 51.5 to $53 \mathrm{hpf}(\mathbf{b}, \mathbf{d}, \mathbf{e}, \mathbf{g}, \mathbf{i}$ and $\mathbf{j})$. Without Epi stimulation, pri-miR-21-1 was expressed only in the valve-forming endocardium (a), not in the head vasculature (f). Elimination of blood cells from the circulation by knocking down gatal did not affect the expression (c,h). After stimulation by Epi, expression of pri-miR-21-1 became slightly stronger at the AV canal (b). (g) Expression in the head became evident in the metencephalic artery/middle cerebral vein (yellow arrowheads), the primary head sinus (green arrowheads) and the primordial mid-brain channel (black arrowheads). This ectopic induction was not affected in the gata1 morphants (i), but the absence of heartbeat cancelled it (j). (k) Relative expression of the mature miR-21 was measured by qRT-PCR using RNA isolated from the whole bodies. In the WT, Epi induced $\sim 1.7$-fold, whereas the induction in the tnnt2 morphant was minimal. In the gata1 morphants, miR-21 was induced at a relatively lower extent than the WT ( $\sim 1.4$-fold). Scale bars, $50 \mu \mathrm{m}(\mathbf{a}-\mathbf{e}), 100 \mu \mathrm{m}(\mathbf{f}-\mathbf{j})$.

stimulation, the expression of pri-miR-21-1 in the valve was slightly intensified (Fig. 3b), relative to the normal expression (Fig. 3a). In the tnnt 2 morphants, the expression was repressed, even in the presence of epinephrine (Fig. 3e). Next, we injected MO against gata1, which eliminated blood cells ${ }^{22}$ and lowered the viscosity ${ }^{7}$. The expression of pri-miR-21-1 was not affected (Fig. 3c) and, in the stimulated heart, this expression was enhanced (Fig. 3d).

Although the endothelial cells in the head do not express primiR-21-1 (Fig. 3f), they exhibited strong expression after the epinephrine treatment (Fig. 3g). This induction was not cancelled by injection of the gata1 MO (Fig. 3i). In the tnnt 2 morphants, pri-miR-21-1 was not expressed in the head, even after the epinephrine stimulation (Fig. 3j). Injection of the gata1 MO alone did not induce pri-miR-21-1 (Fig. 3h).

To confirm further, we semi-quantitated the expression of $m i R$ 21 by quantitative real-time PCR (qRT-PCR) on RNA isolated from the whole bodies (Fig. 3k). The epinephrine treatment induced miR-21 $\sim 1.7$-fold in the WT and 1.4-fold in the gata1 MO. As expected, this induction was cancelled in the tnnt 2 .

Haemodynamic parameters were measured in the middle cerebral vein before and after the adrenergic stimulation (see Supplementary Fig. S2). The shear stress was estimated to be increased 2.1-fold by epinephrine.

Physical regulation of $m i R-21$ expression was confirmed using cultured mammalian cells (see Supplementary Fig. S2). Application of $2.0 \mathrm{~Pa}$ of shear stress (equivalent to that at the luminal surface of the human aorta ${ }^{25}$ ) with a unidirectional laminar flow over human umbilical vein endothelial cells (HUVECs) rapidly induced expression of mature $m i R-21$. The primary $m i R-21$ transcript was induced more strongly. KLF2 was induced rapidly within $15 \mathrm{~min}$, and the expression increased progressively, hitting the highest peak after $2 \mathrm{~h}$. These results indicate that a set of genes can be induced by the shear stress, but with different temporal profiles.
We have found another valve-specific gene, egrl, which expression is dependent on the heartbeat (see Supplementary Fig. S2). Previously, we have reported that myocardial expression of $m i R-143$ in the OFT is dependent on the heartbeat ${ }^{12}$. Nonetheless, induction of $m i R-143$ by mechanical stresses is slower. This suggests that the adaptive responses triggered by the mechanical stimuli are temporally controlled. Laminar flow has been shown to induce other genes in HUVECs (including Klf2, COX-2, ICAM-1 and $c-f o s)^{26,27}$, but the response of $m i R-21$ is the fastest.

In addition to the temporal control, cells react differentially to different flows, such as laminar versus turbulent flows and unidirectional versus reciprocating ones ${ }^{28}$. pri-miR-21 was induced more rapidly when reciprocating flow at $0.5 \mathrm{~Hz}$ was applied, although induction of mature $m i R-21$ was kept weak (see Supplementary Fig. S2). In this condition, Klf2 was also induced faster, but weakly than the unidirectional flow. Induction of pri-miR-21 was sustained longer. $1.0 \mathrm{~Hz}$ reciprocating flow exerted the similar effect, but induction of pri-miR-21was more robust. Interestingly, induction of mature $m i R-21$ was very weak in HUVECs stimulated by the reciprocating flow. This suggests that processing of pri-miR-21 might be repressed by this flow. This also implies that the actions of $m i R-21$ might be repressed even after stimulation by specific shear stress, consistent with the fact that genetic deletion of $m i R-21$ does not affect the correct formation of vasculature and heart ${ }^{15}$.

A valveless phenotype of $\boldsymbol{m i R}-21$ morphants. To explore the roles of $m i R-21$, we injected MOs of several designs against $m i R-21$ at the one-cell stage (Fig. 4) (see Methods). For this experiment, we designed two types of MOs, one for multiblocking that disturbs both Dicer and Drosha-mediated processing and another for Guide Dicer that masks guide sequence to block processing $m i R-21-1$ and $m i R-21-2$ by Dicer. These MOs gave 

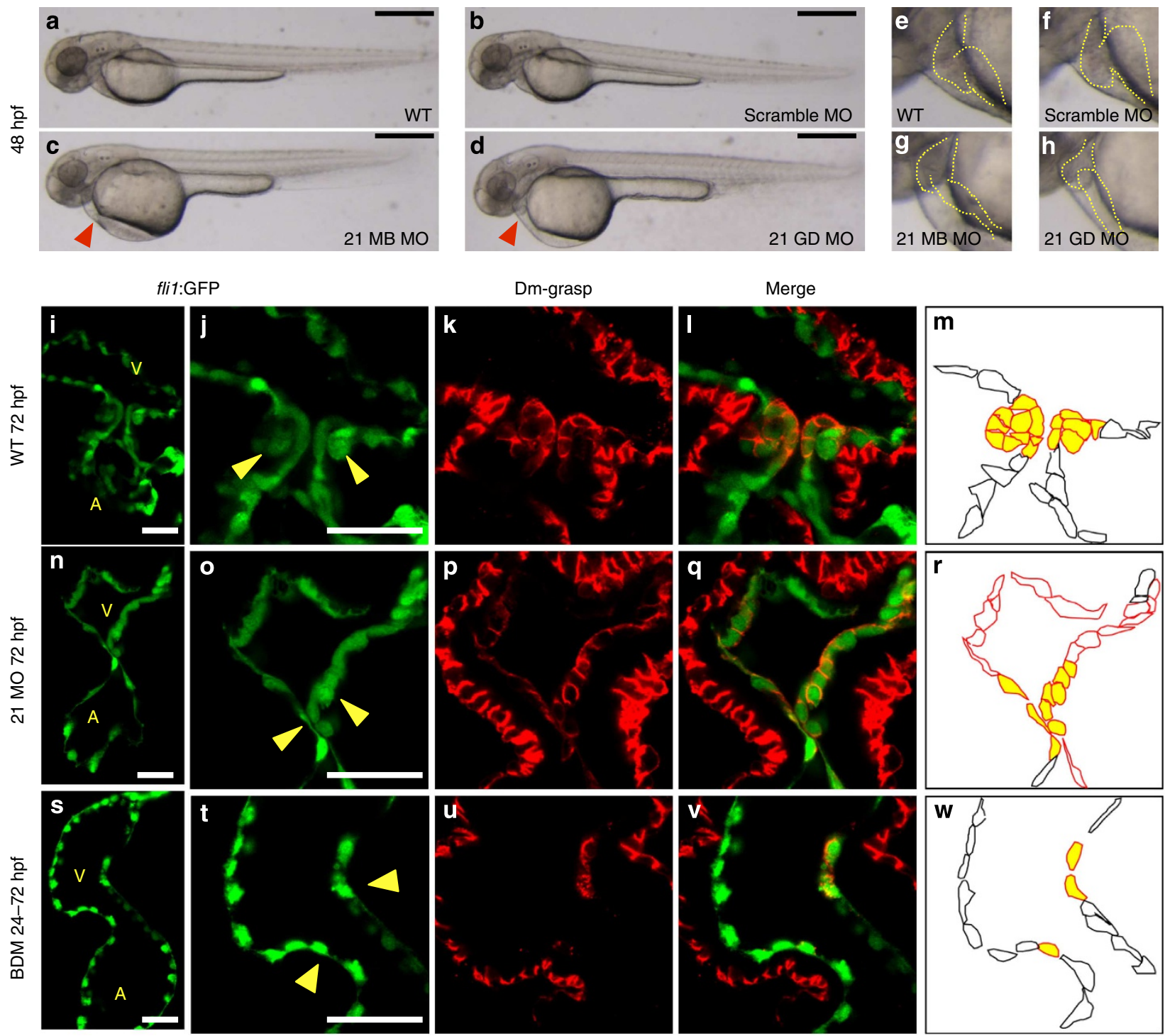

Figure 4 | A valveless miR-21 morphant. (a-d) Blood flow of the miR-21 morphants stagnated with pooling of blood cells on the yolk at $48 \mathrm{hpf}$ (red arrowhead in $\mathbf{c}, \mathbf{d})(\mathrm{MB}$, multiblocking MO; GD, Guide Dicer MO). This stagnation was neither present in the WT (a) nor embryos injected with a scrambled $\mathrm{MO}$ (b). Higher magnification views are shown in $\mathbf{e}-\mathbf{h}$, with heart contours indicated by dotted lines. (i-m) At 72 hpf, thick layers of the GFP-positive endocardial cells were protruded into the AV canal (i), with cuboidal thickening cells (yellow arrowheads in $\mathbf{j}$ ). Cell shapes are visualized by Dm-grasp staining (k,I). Dm-grasp- and GFP-positive cells are cuboidal, whereas Dm-grasp-negative and GFP-positive cells are squamous (I). (m) A schematic representation of the endocardial cells, with cells in the valve-forming area shown in yellow, Dm-grasp-positive cells in red lines, negative cells in black lines, respectively. A, atrium, V, ventricle. (n-r) In the miR-21 morphants, only a thin layer of endocardial cells was evident with neither thickening nor protrusion into the lumen. (s-w) When heartbeat was stopped from 24 to $72 \mathrm{hpf}$ by BDM, neither thickening nor protrusion of the endocardium was initiated. The number of the Dm-grasp-positive endocardial cells was reduced. Scale bars, $500 \mu \mathrm{m}(\mathbf{a}-\mathbf{d}), 50 \mu \mathrm{m}(\mathbf{i}, \mathbf{j}, \mathbf{n}, \mathbf{0}, \mathbf{s}, \mathbf{t})$.

similar results. Co-injection of both miR-21-1 and miR-21-2 MOs knocked down miR-21 more efficiently. The expression of miR-21 in the morphants was reduced to one-third of that in the WT (see Supplementary Fig. S3). Scrambled MO did not induce obvious abnormalities (Fig. 4b). At $48 \mathrm{hpf}$, blood pooled on the surface of the yolk in the morphants indicating stagnation of flow (Fig. $4 \mathrm{c}, \mathrm{d})$. The hearts of the morphants are narrower, showing incomplete looping (Fig. 4g,h). Other parts of body were normal, except for slight hypoplasia of the hindbrain, where $m i R-21$ was weakly expressed.

In sections, the clustering and thickening of the valve-forming cells, visualized by fli1:GFP ${ }^{29}$ and Dm-grasp staining ${ }^{30}$, were absent in the morphants at $72 \mathrm{hpf}$ (Fig. $4 \mathrm{n}-\mathrm{r}$ ), whereas those are obvious in the WT (Fig. 4i-m). These changes were also lost in the hearts arrested from 24 to $72 \mathrm{hpf}$ by BDM (Fig. 4s-w). Although the similar abnormalities in the endocardium were evident at $55 \mathrm{hpf}$, myocardial cell shape change in the AV canal occurred normally in the morphants. (see Supplementary Fig. S4). Further verification was provided by rescue experiments using miR-21 duplex (see Supplementary Fig. S3).

As there were fewer valve-forming cells in the morphants, we counted their numbers, and found significant reductions in both the miR-21 morphants and the BDM-treated hearts, without affecting the myocardial cells (see Supplementary Fig. S3). In addition, expression of proliferating cell nuclear antigen $(\mathrm{PCNA})^{31}$ was lost or weak in the valve-forming cells in the miR-21 morphants and embryos treated with BDM (see Supplementary Fig. S5). 
We examined the expression of several markers. Specification of the atrial versus ventricular identity was not affected (see Supplementary Fig. S3). In addition, expression of two myocardial markers was not affected by the $m i R-21 \mathrm{MO}$, although expression of versican was expanded (see Supplementary Fig. S6). Expression of two markers for the valve-forming endocardial cells, hyaluronan synthase 2 (has2) and notch1b, was observed widely in both the atrial and the ventricular compartments (see Supplementary Fig. S6). Flow-dependent expression of egr1 was severely reduced $^{32}$, probably due to weak blood flow in the miR-21 morphants (see Supplementary Fig. S6). Expression of the secreted phosphoprotein 1 ( $s p p 1)$, the endocardial marker at later stages of the valve development, was reduced, suggesting that maturation of valves was impaired. This decline might be partially due to the weak expression of egrl, as Spp1 was reported to be a direct target of Egr1 in mouse and GC-rich Egr1-binding motifs are conserved in $\operatorname{spp} 1$ (ref. 33). As shown later, a loss of function of $m i R-21$ results in reduction of the mitogen-activated protein (MAP) kinase cascade. As the MAK kinase signals were shown to be involved in the expression of Egrl (ref. 34), this pathway could modulate expression of egr 1 in zebrafish. Expression of $k l f 2 a$ was not affected.

Sprouty 2 as a target of $m i R-21$. As a member of the oncomiRs, miR-21 has been analysed in mammals, and several targets identified $^{14,35}$. Sprouty2 (spry2), which encodes a potent inhibitor of the RTK/Ras/ERK pathway, is a direct target ${ }^{36,37}$. An exhaustive search of three databases (miRbase, RNAi web and BiBiServ) revealed no new targets but confirmed spry 2 in zebrafish. As shown in Fig. 5a, the seed sequence of zebrafish $m i R-21$ shows significant paring at its $5^{\prime}$-end (see Supplementary Fig. S7). To verify further, we synthesized two mRNAs. The first mRNA encoded enhanced green fluorescent protein (EGFP) with a $3^{\prime}$-untranslated region (UTR) of spry 2 and the other encoded mCherry fluorescent protein with a poly(A) signal alone. When these mRNAs were injected along with the miR-21 MO, both green and red fluorescent signals were detected (left panel, Fig. 5b). However, when a synthesized duplex of $m i R-21$ was co-injected, EGFP signals was selectively repressed (middle panel, Fig. 5b), indicating that translation of EGFP was inhibited by the miR-21 duplex. When the seed sequence in the $3^{\prime}$-UTR of spry 2 was mutated, EGFP signals were not repressed (right panel, Fig. 5b).

These results are consistent with observations that although spry2 is not normally expressed in the OFT and AV canal, it was induced in the $m i R-21$ morphants (Fig. $5 \mathrm{c}, \mathrm{g}$ ), indicating that the regulatory action of $m i R-21$ is mediated at least by inhibition of spry 2 and removal of suppression of the MAP kinase cascade. We also confirmed that expression of programmed cell death $4 a$ $(p d c d 4 a), 4 b(p d c d 4 b)$ and phosphatase and tensin homologue $B$ (ptenb) were induced in the $m i R-21$ morphants (Fig. $5 \mathrm{~d}-\mathrm{f}$ and $\mathrm{h}-\mathrm{j}$ ), consistent with mouse $m i R-21$ (refs 38,39 ). Hence, in both species, $m i R-21$ acts as a positive regulator of cell proliferation. Sequence alignments of the target regions are shown in Supplementary Fig. S7. These results were further confirmed by qRT-PCR, using RNA isolated from whole bodies expressing $m i R-21$ as a transgene (see Supplementary Fig. S7).

To confirm the roles of spry2 and the MAP kinase cascade, we injected a target protector MO against spry2 (spry2 target protection (TP) MO). Protection of spry2 was confirmed (see Supplementary Fig. S8). At 48 hpf, the spry2 TP MO-injected embryos showed signs of pericardiac oedema and incomplete looping of the heart tube (Fig. 6b,f). Next, we treated developing embryos with U0126, an inhibitor of the MAP kinase pathway to mimic the inhibitory effect of spry2 (ref. 40). U0126-treated embryos showed a cardiac phenotype similar to that of the spry 2
TP MO-injected embryos (Fig. 6d,h) and $m i R-21$ morphants $(N=92 / 145$ for the spry2 TP morphants and 56/92 for the U0126 treatment) (Fig. 4).

Morphological alterations were confirmed by the green fluorescent protein (GFP) signals and Dm-grasp staining in fli1:GFP transgenic fishes at $72 \mathrm{hpf}$ (Fig. $6 \mathrm{i}-\mathrm{w}$ ). In both the spry2, TP MO-injected and U0126-treated embryos, the endocardial cells showed neither clustering nor thickening, with only a single layer of thin GFP-positive cells (Fig. 6n-w). As revealed by Dmgrasp staining, only a few thin cells were visible at the AV canal (Fig. 6p-r and $u-w$ ), whereas clustering of the multi-layered cuboidal cells was formed in the WT (Fig. 6k-m). We also found that the endocardial cell number at the AV canal was decreased approximately by half in both the spry2 TP MO-injected and U0126-treated embryos (see Supplementary Fig. S8). In both the spry2, TP MO-injected and U0126-treated embryos, the myocardial expression of versican was expanded, whereas bmp4 was unchanged. The endocardial expression of notch $1 b$ and has2 became stronger. Expression of spp1 was suppressed (see Supplementary Fig. S9). PCNA staining was also suppressed (see Supplementary Fig. S5).

Next, we examined MAP kinase signalling by staining a phosphorylated form of Erk1/2 at $55 \mathrm{hpf}$ (Fig. 7). In the WT, staining was evident in the cuboidal endocardial cells at the AV boundary (Fig. $7 \mathrm{a}-\mathrm{c}$ ). In the $m i R-21$ morphants, staining was lost (Fig. $7 \mathrm{~d}-\mathrm{f}$ ). When heartbeat was arrested from 24 to $55 \mathrm{hpf}$ by BDM, faint signals were detected only on the luminal surface (Fig. $7 \mathrm{~g}-\mathrm{i}$ ). Likewise, staining was significantly repressed in the spry2 TP MO-injected and U0126-treated embryos (Fig. 7j-o). These data indicate that the heartbeat, $m i R-21$, spry 2 and MAP kinases are in the same signalling cascade.

\section{Discussion}

Once contraction of cardiomyocytes commences, it continues for the entire lifetime. However, patterns of blood flow change along with transformation of the heart structures. Oxygen and nutritional demands change during development and with physiological and pathological conditions in adults so that cardiac tissues must change their structure and function accordingly. In both cardiogenesis and homoeostatic adaptation, cardiac tissues sense physical forces generated by the heartbeat and blood flow and respond with changes in gene expression. Thus, cardiac cells are mechanotransducers that convert physical inputs into biological outputs ${ }^{41,42}$. The molecular mechanisms of this signal conversion are largely unknown. Functional links between physical forces and gene expression are implied by the demonstration that the lineage of mesenchymal stem cells can be directed by matrix elasticity ${ }^{43}$.

In the vascular system, shear stress induces Klf2. Knock-out of mouse Klf2 results in a loss of the vascular integrity and subsequent haemorrhage, reinforcing the role of shear stress-dependent $K l f 2$ expression in the vascular homoeostasis ${ }^{44}$. In zebrafish, expression of $k l f 2 a$ in the $\mathrm{AV}$ canal is dependent on reversing blood flow. A loss of function of $k l f 2 a$, which can be mimicked by arresting heartbeat or injecting an MO against $k l f 2 a$, results in dysgenesis of the valve ${ }^{7}$. These lines of evidence emphasize that physical stimuli regulate gene expression, but to date, the precise mechanisms remain to be elucidated.

This report has shown that expression of zebrafish $m i R-21$ is dependent on the heartbeat. Arrest of the heart for $12 \mathrm{~h}$ eliminated the expression of both the primary and mature forms of $m i R-21$ (Fig. 2). More importantly, its expression was restored by a period of heartbeat as short as $1 \mathrm{~h}$ (Fig. 2). A similar rapid response was seen in the epinephrine-stimulated head vasculatures (Fig. 3) and HUVECs stimulated by shear stress (see 
z-miR-21 3'- CGGUUGUGGUCAGACUAUUCGAU

z-miR-21 3' UTR (wild type) 5'- UGACAGUGGAUUGGCAAAAACUG z-miR-21 3' UTR (seed deleted) 5'- UGACAGUGGAUUGGCCCAUCGAU

b
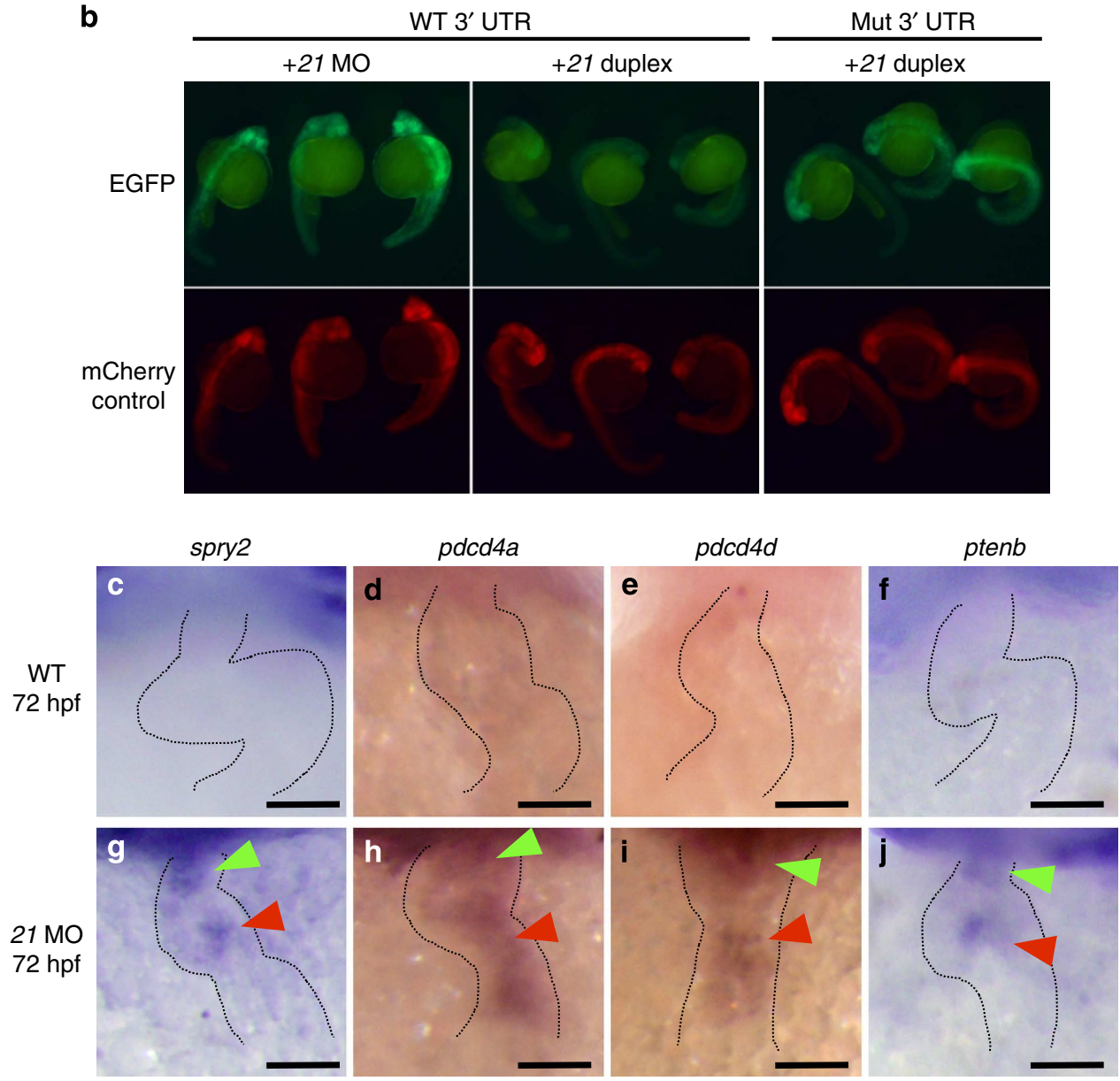

Figure 5 | Sequence alignment and expression patterns of selected genes. (a) Sequence alignment of zebrafish miR-21, $3^{\prime}-U T R$ of spry2 and its mutated version is shown, with the seed sequences highlighted in yellow and the complementary nucleotides in red and green. (b) The EGFP-spry 2 reporter with the WT spry2 3'-UTR gave strong fluorescent signals when the endogenous miR-21 was blocked by the MO against it (left panel), but failed to show the signals when co-injected with the miR-21 duplex (middle panel). The EGFP-spry2 reporter mRNA, in which the seed sequence was mutated, produced strong signals even when co-injected with the miR-21 duplex (right panel) at $24 \mathrm{hpf}$. (c,g) Expression of the endogenous spry2 was not observed in the WT at $72 \mathrm{hpf}(\mathbf{c})$, whereas it was induced in both the OFT and AV canal in the morphant (green and red arrowheads in $\mathbf{g}$, respectively). (d-j) Other target genes, $p d c d 4 a(\mathbf{d}, \mathbf{h}), \operatorname{pdcd} 4 b(\mathbf{e}, \mathbf{i})$ and ptenb $(\mathbf{f}, \mathbf{j})$ were induced in the morphant $(\mathbf{h}-\mathbf{j})$. Expression of these genes was not observed in the WT $(\mathbf{c}-\mathbf{f})$. Scale bars, $50 \mu \mathrm{m}(\mathbf{c}-\mathbf{j})$.

Supplementary Fig. S2). However, the induction profiles of the pri-miR-21, mature $m i R-21, K L F 2$ are different in HUVECs, depending on the natures of the applied flows. Endothelial cells are known to respond differently to chaotic and steady flows ${ }^{45,46}$, and the expression of zebrafish $k l f 2 a$ is dependent on the reversing flow, reinforcing the importance of the flow during cardiogenesis and physiological adaptation.

In addition, a set of genes can be induced by the flow in a temporally controlled manner, with $m i R-21, k l f 2 a$ and egrl as early responders and $m i R-143$ and $m i R-23$ as late ones. In this sense, it is particularly important that $m i R-23 b$ and $m i R-143 / 145$ can be induced by KLF2 in HUVECs ${ }^{47}$, and that EGR1 binds to regulatory regions of the $m i R-23 a / 24 / 27 a$ cluster and $K L F 2$ (ref. 48). This indicates that this hierarchical induction contributes to the distinct temporal profiles of gene induction.
Recently, stress-dependent remodelling of cardiac tissues has been shown to occur in the absence of $m i R-21$ in mouse (T.B. et al., unpublished observation $)^{17,36,49}$. Genetic deletion of miR-21 protects against tumour formation via modulation of the MAP kinase cascade ${ }^{15}$ but the valveless phenotype was not found in either miR-21-null or antagomir-treated mice ${ }^{15,17}$. This implies that $m i R-21$ acts differently in different tissue contexts (cardiomyocytes/cardiac fibroblast in mouse versus valve-forming endocardial cells in zebrafish), yet regulating the same targets. Phenotypic differences are also seen in Klf2/klf2a. Klf2-null mice show abnormal vascular morphology, a loss of vessel wall stability and heart failure ${ }^{44}$, whereas the klf2a-morphants display dysmorphogenesis of the valve ${ }^{7}$. The phenotypic discrepancies observed in $m i R-21$ and $K l f 2 / k l f 2 a$ might relate to the fact that valve leaflets arise from the mesenchyme cushion in mice, 

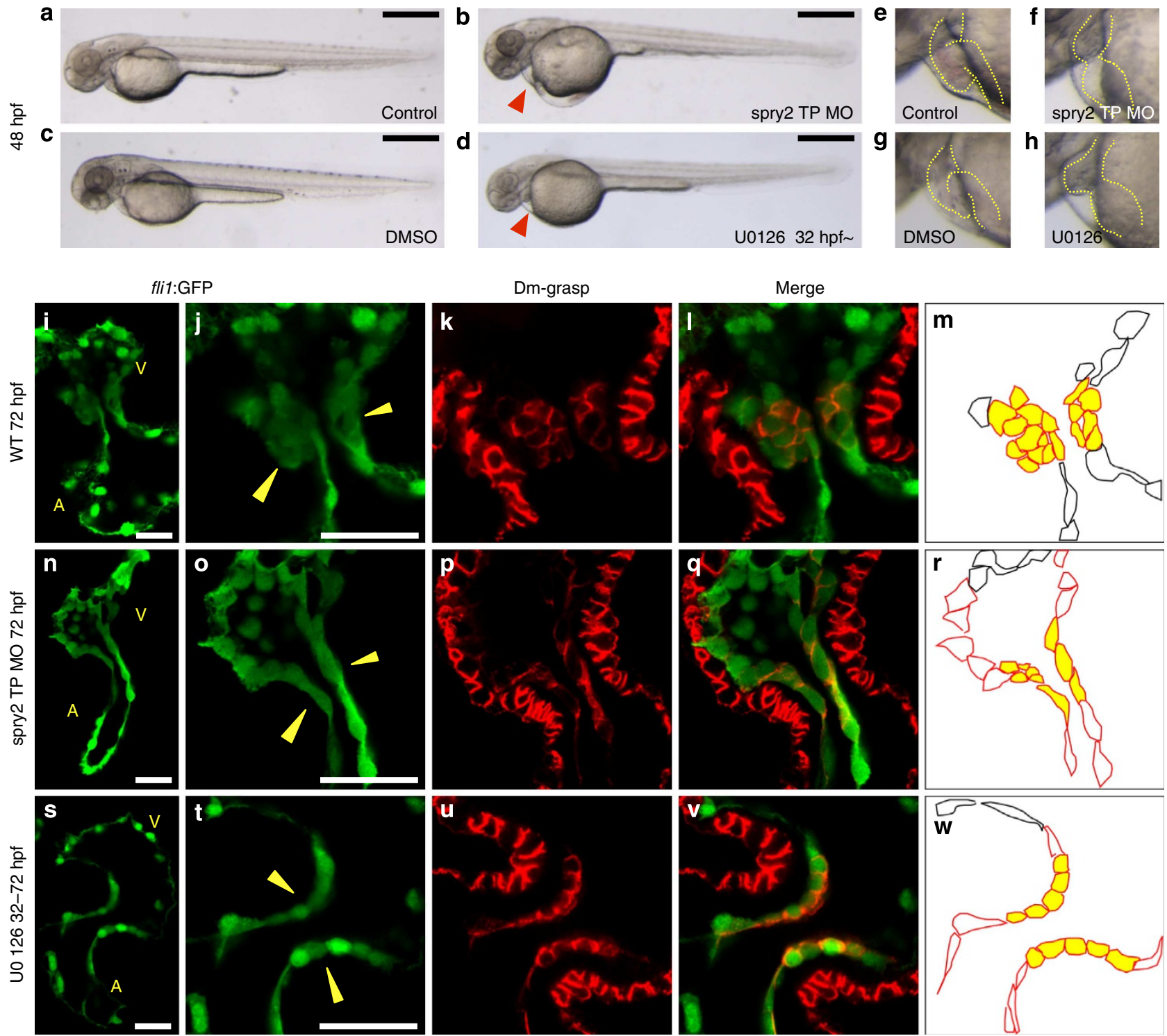

Figure 6 | Effects of a gain of function of spry2 and U0126 treatment. (a-h) When the MAP kinase cascade was blocked by injection of a target protection (TP) MO of spry2 (b,f) or U0126-treatment (d,h), hearts (dotted outlines) exhibited incomplete looping (f,h). Red arrowhead indicates pooling of blood, a sign of stagnation of circulation. The normal looped heart is shown in (e). (i-w) As observed in the miR-21 morphants and the arrested hearts, the endocardial cells were single-layered, flattened and stretched in the hearts injected with the TP MO of spry2 (n-r) and the U0126-treated embryos (s-w). Normal thickening of cells observed in the WT (i-m) was lost, as visualized by flit-GFP reporter and Dm-grasp staining. As illustrated in a schematic representation ( $\mathbf{m}, \mathbf{r}$ and $\mathbf{w}$ ), the multi-layered cuboidal cells observed in the WT (m) was detected neither in the hearts injected with the target protection MO of spry2 (r) or treated by U0126 (w). Scale bars, $500 \mu \mathrm{m}(\mathbf{a}-\mathbf{d}), 50 \mu \mathrm{m}(\mathbf{i}, \mathbf{j}, \mathbf{n}, \mathbf{0}, \mathbf{s}, \mathbf{t})$.

whereas they are formed by protrusion of the endocardium in zebrafish $^{50}$. As cardiomyocytes and mesenchymal cells do not contact the blood stream, they are unable to receive flow stimuli. This spatial separation prevents them from responding to shear stress. Shear stress and stretch activate $m i R-21$ with different temporal profiles (our unpublished observation), hereby these two stimuli could act differently. In addition, signalling cascades specifically acting on cardiomyocytes, such as BMP, might modify the actions of $m i R-21$, as suggested by SMAD-dependent maturation of miR-21 (ref. 51). We also do not exclude the possibility that myocardial contraction might affect expression of $m i R-21$ by an unknown mechanism.

In addition to these developmental contexts, the haemodynamic forces may differ between the zebrafish and mouse; for example, the Reynolds number at the valve-forming stage is 0.017 at $26 \mathrm{hpf}$ in zebrafish and 0.205 at $10.5 \mathrm{dpc}$ in the mouse $\mathrm{s}^{52}$, implying that viscous shear forces are relatively more important for zebrafish cells.

$m i R-21$ is also expressed in the OFT and AV valves of adult hearts (Fig. 1). In adult mammals, valves and vascular endothelial cells are susceptible to mechanical injury and pathogenic conditions associated with irregular shear, leading to cell proliferation and expression of genes, including Egr1 (refs 53-55). Accordingly, the expression miR-21 in the adult valves of zebrafish might be involved in their homoeostatic maintenance.

Recently, it was shown that excessive endocardial cushions were formed in zebrafish dicer mutant ${ }^{30}$, a conflict result with the miR-21 morphants. However, in the dicer mutant, processing of all miRNAs are blocked not only in the endocardium, but also in the myocardium, implying that unknown miRNA(s) might be negatively involved in the formation of endocardial cushions. 

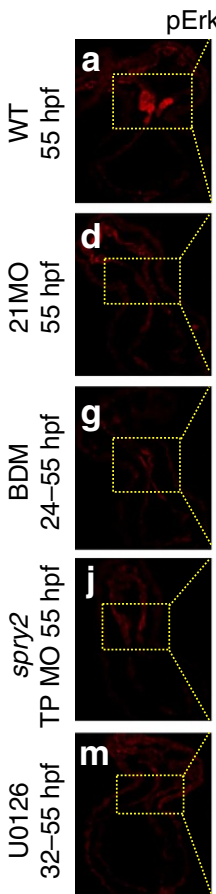

Erk1/2
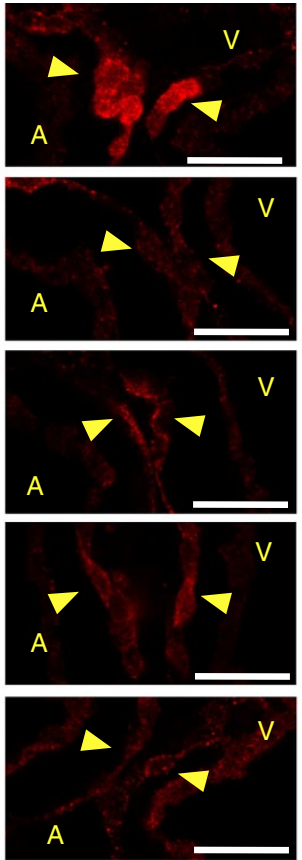

fli1:GFP
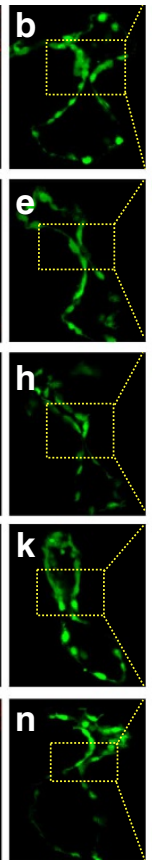
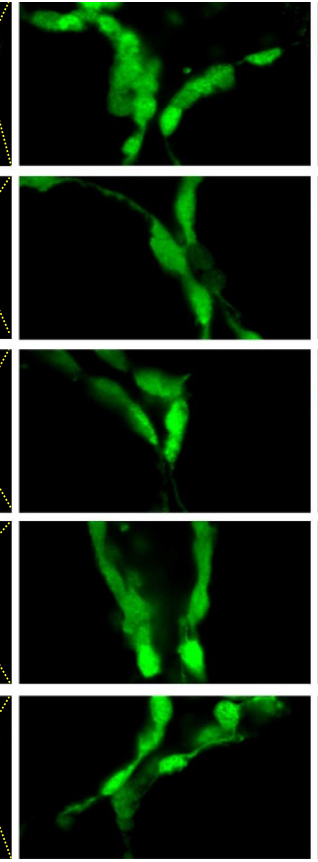

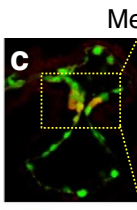

Merge
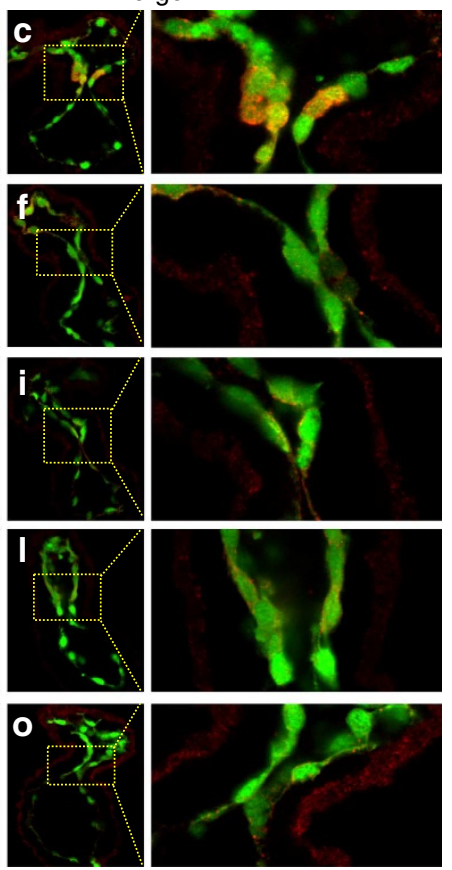

Figure 7 | Suppression of MAP kinase signalling in miR-21 morphants and BDM-treated hearts. (a-c) In the WT beating hearts, a phosphorylated form

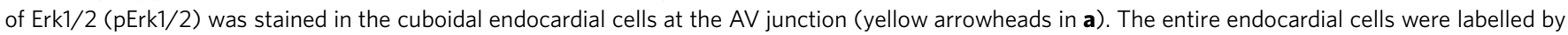
GFP, which expression was driven by the fli1 promoter $(\mathbf{b}, \mathbf{c})$. (d-f) In the miR-21 morphants, the pErk1/2 staining was week and the cells were flat. ( $\mathbf{g}-\mathbf{i})$ When the heartbeat was stopped by BDM, phosphorylation of Erk1/2 was also suppressed, leaving only faint staining in the apical surface of flattened endocardial cells. (j-o) Injection of the miR-21 target protection MO and the U0126 treatment gave the same results on pErk1/s staining. A, atrium; $\mathrm{V}$, ventricle. Scale bars, $20 \mu \mathrm{m}$.

\section{Table 1 | qRT-PCR primers.}

\section{Forward}

z-miR-21 5'-TAGCTTATCAGACTGGTGTTGGC-3'

h-miR-21 5'-TAGCTTATCAGACTGATGTTGA-3'

h-pri-miR-21 5'-TTTTGTTTTGCTTGGGAGGA-3'

$z-k l f 2 a 5^{\prime}$-CACACAGGCGAGAAGCCCTA-3'

h-KLF2 5'-CAAGACCTACACCAAGAGTTCG-3'

z-egr1 5'-TCAGACCTACTCCACCCAACG-3'

z-spry2 5'-AGCCTTGGGATGGTCAGGTG-3'

z-ptenb $5^{\prime}$-GCAGAGTTGCACAGTATCCATTTGA-3'

z-pdcd4a 5'-GGTCTATGAGGCTGTGGTTATGG-3'

$z$-pdcd4b 5'-TCAGATGAGACGGGGCTTTG-3'

$z$-notch1b 5'-AATCCCCGTGCAGAAATGGT-3'

$z$-has2 5'-TGATTCAGACACCATGCTGGA-3'

$z$-bmp4 5'-CCTGGAGGAGCTGCAGTCAG-3'

z-versican 5'-TGCGCATTAAGTGGACCAGA-3'

$z$-miR-23 5'-ATCACATTGCCAGGGATTTCCA-3'

z-miR-24 5'-TGGCTCAGTTCAGCAGGAACAG-3'

z-miR126 5'-TCGTACCGTGAGTAATAATGC-3'

z-miR-138 5'-AGCTGGTGTTGTGAATCAGGCC-3'

$z$-miR-143 5'-TGAGATGAAGCACTGTAGCTC-3'

z-miR-145 5'-GTCCAGTTTTCCCAGGAATCCC-3'

z-miR-150 5'-TCTCCCAATCCTTGTACCAGTG-3'

z-ef1 alpha 5'-CTGTGGCAACAGGTGCAGTTCTA-3',

h-EF1 alpha 5'-TGCGGTTTTTGTCATCAAA-3'

\section{Reverse}

h-pri-miR-21 5'-AGCAGACAGTCAGGCAGGAT-3' z-klf2a $5^{\prime}$-GAGATGGTCGGACCTGGAGA-3' h-KLF2 5'-CATGTGCCGTTTCATGTGC-3' z-egr1 5'-CCAGGCTGAACAGAGGCTCA-3' z-spry2 $5^{\prime}$-TTCCTATGCTCGGGCAGCTC-3' z-ptenb $5^{\prime}$-TCACTCCCTTCTTGTCTCTGGTCC-3' $z$-pdcd4a $5^{\prime}$-CCCCTGTTCATCTGGTCCAA-3' $z-p d c d 4 b 5^{\prime}$ - CTTACGGCCCCGACTAGGAC- $3^{\prime}$ z-notch1b 5'-CACGTTGTTGGTGGGAGTGA-3' z-has2 5'-ACCTCACGCTGCTCAGGAAG-3' z-bmp4 5'-GTTCGTCCTCTGGGATGCTG-3' z-versican 5'-GACTCCTGTAGCCCGAACCA-3' z-ef1 alpha 5'-CGCAGGAAAGGAAAACAACTTGG-3' h-EF1 alpha 5'-AAGAGTGGGGTGGCAGGTATTAG-3'

Results in Supplementary Fig. S3, Supplementary Figs. S1-S3 and Supplementary Fig. S7 are shown as mean fold changes \pm s.e.m.

We have demonstrated that $m i R-21$ is a novel component of a mechanotransduction pathway in which blood flow is the primary stimulus for gene expression. This is summarized in Supplementary Fig. S10. In effect, physical forces act as a 'morphogen' producing a gradient of mechanical strain in tissues.
Anisotropic distribution of strains is translated into asymmetric gene expression. Future research will look for factors shuttling between the cytoplasm and nucleus in response to mechanical stresses, that is, potential regulatory factors of $m i R-21$. By integrating these lines of investigation, we aim to achieve a 
better understanding of the physical aspects of cardiogenesis and circulatory homoeostasis and, ultimately, to develop new therapeutic methods for diseases caused by haemodynamic changes.

\begin{abstract}
Methods
Antibodies and reagents. To change the haemodynamics, zebrafish embryos were placed in water containing $10 \mathrm{mM}$ of BDM (2,3-butanedione monoxime: Sigma, B-0753) or $2 \mathrm{mM}$ of epinephrine (Wako) at proper stages. For exposure to U0126 (Merck Ltd), embryos were incubated with $100 \mu \mathrm{M}$ U0126 in 2\% dimethyl sulfoxide from $32 \mathrm{hpf}$ until fixation. In all experiments, embryos treated with the vehicles alone were used as a control. An antibody against pERK1/2 (1:100) was purchased from Cell Signaling Technology. Antibodies against dm-grasp (1:200), GFP (1:500) and zebrafish PCNA (1:100) were from Developmental Studies Hybridoma Bank (DSHB), Sigma and AnaSpec, respectively.
\end{abstract}

cDNA probes and in situ hybridization. Zebrafish in situ hybridization probes were isolated from complementary DNA (cDNA) templates using standard PCR techniques with appropriate sets of primers. To detect expression of mature miR21, DIG-labelled LNA probes were purchased from Integrated DNA technologies. Both whole mount and section in situ hybridization experiments were performed according to the protocol published by Exiqon ${ }^{56,57}$.

Quantitative real-time PCR. Approximately 20 zebrafish whole embryos, 10-15 zebrafish hearts and cultured cells (approximately $1 \times 10^{5}$ HUVECs) were homogenized with MULTI-BEADS SHOCKER (MB901U, Yasui Kikai, Osaka, Japan). Total RNAs were isolated by RNeasy Kit (Qiagen), according to the manufacturer's instructions. Each sample was reverse-transcribed into cDNA by miScript RT Kit (Qiagen) and was subjected to qRT-PCR analysis with miScript SYBR Green PCR Kit (Qiagen). Each experiment was carried out with at least one additional biological and experimental replicate. Results in Fig. 3 and Supplementary Fig. S1-S3 are shown as mean fold changes \pm s.e.m. qRT-PCR primers shown in Table 1.

Immunohistochemistry and cell counting. Zebrafish embryonic hearts were isolated in L-15 medium (Gibco) and fixed in 4\% paraformaldehyde for overnight at $4{ }^{\circ} \mathrm{C}$ (for PCNA detection, $1 \mathrm{~h}$ at room temperature). Fixation solution was replaced with PBDT ( $1 \%$ bovine serum albumin (BSA); $0.1 \%$ Triton X-100; $1 \%$ dimethyl sulfoxide in phosphate buffered saline (PBS)) for $1 \mathrm{~h}$. Fixed hearts were incubated with a primary antibody for overnight at $4{ }^{\circ} \mathrm{C}$, briefly washed with PBDT and subsequently incubated with a second antibody conjugated with Alexa Fluor 488 or 594 and 4',6-diamidino-2-phenylindole (Sigma). Hearts were imaged using a confocal microscope (Olympus FV1000). In order to count the cell number, cell counter plugin with ImageJ was employed. For myocardial cells counting, the hspGFF3A transgenic line, which expresses myocardium-specific GFP signal, was used. fli1:GFP transgenic line was used for counting the endocardial cells, as well as the PCNA and/or dm-grasp-positive cells. Error bars express mean \pm s.e.m. and statistical significance was determined by a $t$-test $(P<0.05)$. The transgenic zebrafish lines fli1-EGFP were kindly provided by Kawahara and colleagues ${ }^{58}$. $h s p G F F 3 A$ was described previously ${ }^{59}$.

Injection of morpholino oligonucleotides and mRNAs into zebrafish eggs. MOs were designed and synthesized by Gene Tools (Philomath, OR). Initially, we made several MOs against $m i R-21$. One MO (5'-GCCAACACCAGTCTGATAAG CTA $-3^{\prime}$ ), which was also used by Bakkers and colleagues ${ }^{30}$, was found to be ineffective, as injection of this MO did not induce any specific abnormality in the heart. Other MOs gave the consistent results. MOs were dissolved in the Danieau solution. Approximately $1 \mathrm{nl}$ of MOs were injected into the yolk at the one-cell stage. Sequences, injection concentration and dose are as follows:

miR-21-1, Multiblocking 5'-TGTAACAGCCAACACCAGTCTGATAAGCTA $\mathrm{T}-3^{\prime}(1 \mathrm{mM}, 10 \mathrm{ng})$

miR-21-2, Multiblocking 5'-ACTCAAAGCCAACACCAGTCTGATAAGCTA

C-3' (1 mM, $10 \mathrm{ng})$

miR-21-1, Guide Dicer, 5'-ACAGCCAACACCAGTCTGATAAGCT-3'

(2 mM, 16 ng)

miR-21-2, Guide Dicer, 5'-AAAGCCAACACCAGTCTGATAAGCT-3'

( $2 \mathrm{mM}, 16 \mathrm{ng})$

spry2 miR-21 target protector, $5^{\prime}$-ACTTCAAACCAGTTTTTGCCAATCC-3' (2 $\mathrm{mM}, 16 \mathrm{ng})$

scrambled MO, Oligo-25N (2 mM, $16 \mathrm{ng})$.

Sequences of klf2a, $5^{\prime}$-AGCTGAGATGCATGGACCTGTCCAG-3' (2 mM, $16 \mathrm{ng})$, tnnt2, 5' -CATGTTTGCTCTGATCTGACACGCA-3' (0.5 mM, $4 \mathrm{ng})$, gata1, $5^{\prime}$-CTGCAAGTGTAGTATTGAAGATGTC-3' $(1 \mathrm{mM}, 8 \mathrm{ng})$, gata2, $5^{\prime}$-CATCTAC TCACCAGTCTGCGCTTTG- $3^{\prime}(1 \mathrm{mM}, 8 \mathrm{ng})$, and $t b x 2 b, 5^{\prime}$-GAGCGTGGAAAG GGTGGTAAGCCAT- $3^{\prime}(0.25 \mathrm{mM}, 2 \mathrm{ng})$ were previously described $7,19,22,24$, respectively. To ensure the efficient knockdown, $m i R-21-1$ and $m i R-21-2 \mathrm{MOs}$ were co-injected after mixing equal amounts of two MOs. The miR-21 morphants shown in this article are by the miR-21 multiblocking MO, except for Fig. 4d. For overexpression of $m i R-21$, miRNA duplexes were purchased from Integrated DNA Technologies, Inc., and were resuspended in the Danieau

buffer to a concentration of $100 \mu \mathrm{M}$. The sequences are as follows:

miR-21 duplex A: $5^{\prime}$ - UAGCUUAUCAGACUGGUGUUGGC- $3^{\prime}$

$m i R-21$ duplex B: 5' - GCCAACACCAGUCUGAUAAGCUA-3'.

In vitro transcription of EGFP-z-spry2 $3^{\prime}$-UTR and mCherry mRNAs were performed with mMESSGAE mMACHINE kit (Applied Biosystems) and these synthesized mRNAs were purified with G-50 Micro Columns (GE Healthcare).

Generation of transgenic zebrafish lines. For construction of the miR-21 expression vector, pre-miR-21 sequence was amplified by PCR from CDNA template and cloned into pT2KXIG $\Delta$ in vector ${ }^{60}$. The Tol2 transposon-mediated transgenesis was carried out by following the procedure described elsewhere ${ }^{60}$.

Luciferase assay. HEK293T cells are transfected with psi-CHECK2 vector (Promega) harbouring $z$-miR-21-binding site and the $z$-miR-21/GFP expression vector (pT2KXIG $\Delta$ in-miR-21/GFP). miRNA dual luciferase assay was carried out by using the Dual luciferase assay kit (Promega).

Shear stress experiment. HUVECs were cultured with Medium 199 (Invitrogen) containing 20\% fetal bovine serum (FBS) (SAFC Biosciences), $10 \mathrm{ng} \mathrm{ml}^{-1}$ basic fibroblast growth factor (bFGF) (Austral Biologicals) and $1 \%$ penicillin-

streptomycin (Invitrogen). For experiments, HUVECs were cultured in a $35-\mathrm{mm}$ plastic dish (Iwaki) pre-coated with $0.1 \%$ bovine gelatin (Sigma-Aldrich). The experiments were conducted after the cells reach confluence. For the experiments, medium was replaced with Medium 199 containing 0.5\% FBS and 1\% penicillinstreptomycin, and HUVECs were incubated for $2 \mathrm{~h}$ under static condition. For exposing HUVECs to laminar shear stress, a parallel plate flow-chamber, roller pump (HV-07523-80, Master Flex), two reservoir bottles and silicone tubes were used. One reservoir was open to the atmosphere, and the medium in the flow circuit was equilibrated with $95 \%$ air and $5 \% \mathrm{CO}_{2}$ to maintain $\mathrm{pH}$. For the reciprocating flow experiments, the flow-exposure system consisting of the flowchamber, a 50-ml syringe (Terumo) mounted on a syringe pump (KDS270, KD Scientific), a reservoir glass tube, and silicone tubes were. The flow-chamber was composed of a cell culture dish, a polycarbonate I/O unit, and a silicone gasket. The systems were maintained at $37 \mathrm{C}$. The flow channel with a length of $22 \mathrm{~mm}$, a width of $14 \mathrm{~mm}$ and a height of $0.4 \mathrm{~mm}$ was used for generating shear stress of $2.0 \mathrm{~Pa}$. HUVECs were exposed to laminar flow with a shear stress of $2.0 \mathrm{~Pa}$ or reciprocating flow with a shear stress of $\pm 2.0 \mathrm{~Pa}$ and a frequency of $0.5 \mathrm{~Hz}$ for $15,30,45$, 60,120 and $240 \mathrm{~min}$ in the flow-chamber.

Shear stress estimation. The fli1a:GFP/gata1:RFP transgenic zebrafish line was mounted in low-melting agarose and treated with $2 \mathrm{mM}$ epinephrine, and movies of blood circulation were taken before and $45 \mathrm{~min}$ after epinephrine stimulation with a high speed video camera (EX-F1, CASIO) (30 fps). Velocity of blood flow $(v)$ and vessel diameters were measured by the ImageJ software (15 embryos on average). Velocity of blood flow was estimated from migration speed of three RFPmarked blood cells' centroid in two frames. Average vessel diameter $(a)$ was obtained from three different points of GFP marked-vessel endothelial cells. The shear stress $(\tau)$ is $\tau=4 \mu \mathrm{Q} / \pi a^{3}$. $Q$ is the volumetric flow rate and we postulated that $Q$ is equal to $v$ in this estimation. We assumed that blood viscosity $(\mu)$ is not altered in the presence of epinephrine. Hence, shear stress ratio of epinephrine treated embryos $\left(\tau_{45}\right)$ to untreated $\left(\tau_{0}\right)$ can be given by $\tau_{45} / \tau_{0}$. Error bars represent mean \pm s.e.m. Statistical significance was determined by a $t$-test $(P<0.05)$.

\section{References}

1. Srivastava, D. Making or breaking the heart: from lineage determination to morphogenesis. Cell 126, 1037-1048 (2006).

2. Stainier, D. Y. Zebrafish genetics and vertebrate heart formation. Nat. Rev. Genet. 2, 39-48 (2001).

3. Forouhar, A. S. et al. The embryonic vertebrate heart tube is a dynamic suction pump. Science 312, 751-753 (2006).

4. Hove, J. R. et al. Intracardiac fluid forces are an essential epigenetic factor for embryonic cardiogenesis. Nature 421, 172-177 (2003).

5. Combs, M. D. \& Yutzey, K. E. Heart valve development: regulatory networks in development and disease. Circ. Res. 105, 408-421 (2009).

6. Auman, H. J. et al. Functional modulation of cardiac form through regionally confined cell shape changes. PLoS Biol. 5, e53 (2007).

7. Vermot, J. et al. Reversing blood flows act through klf2a to ensure normal valvulogenesis in the developing heart. PLoS Biol. 7, el000246 (2009).

8. Chen, K. \& Rajewsky, N. The evolution of gene regulation by transcription factors and microRNAs. Nat. Rev. Genet. 8, 93-103 (2007).

9. Jackson, R. J. \& Standart, N. How do microRNAs regulate gene expression? Sci. STKE. 2007, re1 (2007).

10. van Rooij, E. et al. A signature pattern of stress-responsive microRNAs that can evoke cardiac hypertrophy and heart failure. Proc. Natl Acad. Sci. USA 103, 18255-18260 (2006). 
11. Mohamed, J. S., Lopez, M. A. \& Boriek, A. M. Mechanical stretch up-regulates microRNA-26a and induces human airway smooth muscle hypertrophy by suppressing glycogen synthase kinase-3beta. J. Biol. Chem. 285, 29336-29347 (2010).

12. Miyasaka, K. Y. et al. Heartbeat regulates cardiogenesis by suppressing retinoic acid signaling via expression of miR-143. Mech. Dev 128, 18-28 (2011).

13. Nicoli, S. et al. MicroRNA-mediated integration of haemodynamics and Vegf signalling during angiogenesis. Nature 464, 1196-1200 (2010).

14. Selcuklu, S. D., Donoghue, M. T. \& Spillane, C. miR-21 as a key regulator of oncogenic processes. Biochem. Soc. Trans. 37, 918-925 (2009).

15. Hatley, M. E. et al. Modulation of K-Ras-dependent lung tumorigenesis by MicroRNA-21. Cancer Cell 18, 282-293 (2010).

16. Cheng, Y. \& Zhang, C. MicroRNA-21 in cardiovascular disease. J. Cardiovasc. Transl. Res. 3, 251-255 (2010).

17. Patrick, D. M. et al. Stress-dependent cardiac remodeling occurs in the absence of microRNA-21 in mice. J. Clin. Invest. 120, 3912-3916 (2010).

18. Bartman, T. et al. Early myocardial function affects endocardial cushion development in zebrafish. PLoS Biol. 2, E129 (2004).

19. Sehnert, A. J. et al. Cardiac troponin T is essential in sarcomere assembly and cardiac contractility. Nat. Genet. 31, 106-110 (2002).

20. Ostap, E. M. 2,3-Butanedione monoxime (BDM) as a myosin inhibitor. J. Muscle. Res. Cell. Motil. 23, 305-308 (2002).

21. Liu, N. \& Olson, E. N. MicroRNA regulatory networks in cardiovascular development. Dev. Cell. 18, 510-525 (2010).

22. Galloway, J. L., Wingert, R. A., Thisse, C., Thisse, B. \& Zon, L. I. Loss of gata1 but not gata2 converts erythropoiesis to myelopoiesis in zebrafish embryos. Dev. Cell. 8, 109-116 (2005).

23. Zhu, C. et al. Evaluation and application of modularly assembled zinc-finger nucleases in zebrafish. Development 138, 4555-4564 (2011).

24. Chi, N. C. et al. Foxn 4 directly regulates tbx $2 \mathrm{~b}$ expression and atrioventricular canal formation. Genes Dev. 22, 734-739 (2008).

25. Barakat, A. I. A model for shear stress-induced deformation of a flow sensor on the surface of vascular endothelial cells. J. Theor. Biol. 210, 221-236 (2001).

26. Ando, J. \& Yamamoto, K. Effects of shear stress and stretch on endothelial function. Antioxid. Redox. Signal. 15, 1389-1403 (2011).

27. Li, Y. S., Haga, J. H. \& Chien, S. Molecular basis of the effects of shear stress on vascular endothelial cells. J. Biomech. 38, 1949-1971 (2005).

28. Fisher, A. B., Chien, S., Barakat, A. I. \& Nerem, R. M. Endothelial cellular response to altered shear stress. Am. J. Physiol. Lung. Cell. Mol. Physiol. 281, L529-L533 (2001)

29. Lawson, N. D. \& Weinstein, B. M. In vivo imaging of embryonic vascular development using transgenic zebrafish. Dev. Biol. 248, 307-318 (2002).

30. Lagendijk, A. K., Goumans, M. J., Burkhard, S. B. \& Bakkers, J. MicroRNA-23 restricts cardiac valve formation by inhibiting Has 2 and extracellular hyaluronic acid production. Circ. Res. 109, 649-657 (2011).

31. Hurlstone, A. F. et al. The Wnt/beta-catenin pathway regulates cardiac valve formation. Nature 425, 633-637 (2003).

32. Khachigian, L. M. et al. Egr-1 is activated in endothelial cells exposed to fluid shear stress and interacts with a novel shear-stress-response element in the PDGF A-chain promoter. Arterioscler. Thromb. Vasc. Biol. 17, 2280-2286 (1997).

33. Peal, D. S., Burns, C. G., Macrae, C. A. \& Milan, D. Chondroitin sulfate expression is required for cardiac atrioventricular canal formation. Dev. Dyn. 238, 3103-3110 (2009).

34. Schwachtgen, J. L., Houston, P., Campbell, C., Sukhatme, V. \& Braddock, M. Fluid shear stress activation of egr-1 transcription in cultured human endothelial and epithelial cells is mediated via the extracellular signal-related kinase $1 / 2$ mitogenactivated protein kinase pathway. J. Clin. Invest. 101, 2540-2549 (1998).

35. Krichevsky, A. M. \& Gabriely, G. miR-21: a small multi-faceted RNA. J. Cell. Mol. Med. 13, 39-53 (2009).

36. Thum, T. et al. MicroRNA-21 contributes to myocardial disease by stimulating MAP kinase signalling in fibroblasts. Nature 456, 980-984 (2008).

37. Sayed, D. et al. MicroRNA-21 targets Sprouty2 and promotes cellular outgrowths. Mol. Biol. Cell. 19, 3272-3282 (2008).

38. Asangani, I. A. et al. MicroRNA-21 (miR-21) post-transcriptionally downregulates tumor suppressor Pdcd 4 and stimulates invasion, intravasation and metastasis in colorectal cancer. Oncogene 27, 2128-2136 (2008).

39. Roy, S. et al. MicroRNA expression in response to murine myocardial infarction: miR-21 regulates fibroblast metalloprotease- 2 via phosphatase and tensin homologue. Cardiovasc. Res. 82, 21-29 (2009).

40. Hawkins, T. A., Cavodeassi, F., Erdelyi, F., Szabo, G. \& Lele, Z. The small molecule Mek1/2 inhibitor U0126 disrupts the chordamesoderm to notochord transition in zebrafish. BMC. Dev. Biol. 8, 42 (2008).

41. Kruger, M. \& Linke, W. A. Titin-based mechanical signalling in normal and failing myocardium. J. Mol. Cell. Cardiol. 46, 490-498 (2009).

42. Sawada, Y. et al. Force sensing by mechanical extension of the Src family kinase substrate p130Cas. Cell 127, 1015-1026 (2006).

43. Engler, A. J., Sen, S., Sweeney, H. L. \& Discher, D. E. Matrix elasticity directs stem cell lineage specification. Cell 126, 677-689 (2006).
44. Lee, J. S. et al. Klf2 is an essential regulator of vascular hemodynamic forces in vivo. Dev. Cell. 11, 845-857 (2006).

45. Sorescu, G. P. et al. Bone morphogenic protein 4 produced in endothelial cells by oscillatory shear stress stimulates an inflammatory response. J. Biol. Chem. 278, 31128-31135 (2003).

46. Hastings, N. E., Simmers, M. B., McDonald, O. G., Wamhoff, B. R. \& Blackman, B. R. Atherosclerosis-prone hemodynamics differentially regulates endothelial and smooth muscle cell phenotypes and promotes proinflammatory priming. Am. J. Physiol. Cell Physiol. 293, C1824-C1833 (2007).

47. Hergenreider, E. et al. Atheroprotective communication between endothelial cells and smooth muscle cells through miRNAs. Nat. Cell Biol. 14, 249-256 (2012).

48. Tang, C. et al. Global analysis of in vivo EGR1-binding sites in erythroleukemia cell using chromatin immunoprecipitation and massively parallel sequencing. Electrophoresis 31, 2936-2943 (2010).

49. Thum, T. et al. Comparison of different miR-21 inhibitor chemistries in a cardiac disease model. J. Clin. Invest. author reply 31, 462-463 (2011).

50. Scherz, P. J., Huisken, J., Sahai-Hernandez, P. \& Stainier, D. Y. High-speed imaging of developing heart valves reveals interplay of morphogenesis and function. Development 135, 1179-1187 (2008).

51. Davis, B. N., Hilyard, A. C., Lagna, G. \& Hata, A. SMAD proteins control DROSHA-mediated microRNA maturation. Nature 454, 56-61 (2008).

52. Santhanakrishnan, A. \& Miller, L. A. Fluid dynamics of heart development. Cell. Biochem. Biophys. 61, 1-22 (2011).

53. Durbin, A. D. \& Gotlieb, A. I. Advances towards understanding heart valve response to injury. Cardiovasc. Pathol. 11, 69-77 (2002).

54. Geiger, B., Spatz, J. P. \& Bershadsky, A. D. Environmental sensing through focal adhesions. Nat. Rev. Mol. Cell. Biol. 10, 21-33 (2009).

55. Nagel, T., Resnick, N., Dewey, Jr. C. F. \& Gimbrone, Jr. M. A. Vascular endothelial cells respond to spatial gradients in fluid shear stress by enhanced activation of transcription factors. Arterioscler. Thromb. Vasc. Biol. 19, 1825-1834 (1999).

56. Miyasaka, K. Y., Kida, Y. S., Sato, T., Minami, M. \& Ogura, T. Csrp1 regulates dynamic cell movements of the mesendoderm and cardiac mesoderm through interactions with Dishevelled and Diversin. Proc. Natl Acad. Sci. USA 104, 11274-11279 (2007).

57. Wienholds, E. et al. MicroRNA expression in zebrafish embryonic development. Science 309, 310-311 (2005).

58. Fukui, H., Hanaoka, R. \& Kawahara, A. Noncanonical activity of seryl-tRNA synthetase is involved in vascular development. Circ. Res. 104, 1253-1259 (2009).

59. Asakawa, K. et al. Genetic dissection of neural circuits by Tol2 transposonmediated Gal4 gene and enhancer trapping in zebrafish. Proc. Natl Acad. Sci. USA 105, 1255-1260 (2008).

60. Urasaki, A., Morvan, G. \& Kawakami, K. Functional dissection of the Tol2 transposable element identified the minimal cis-sequence and a highly repetitive sequence in the subterminal region essential for transposition. Genetics 174, 639-649 (2006).

\section{Acknowledgements}

We thank S. Takashima and A. Kawahara for supplying materials. This work was supported by a Grant-in-Aid for Scientific Research on Innovative Areas from the Ministry of Education, Science, Sports and Culture of Japan (to T.O.), and grants from the Takeda Science Foundation, the Naito Foundation and the Uehara Memorial Foundation (to T.O.). J.G. was supported by the Tohoku University Cooperative Laboratory Study Program (COLABS). T.B. was supported by Japan Society for the Promotion of Science.

\section{Author contributions}

T.B., J.G., H.O., K.Y.M., Y.S.K. and T.O. designed the study and analysed the data. T.O. wrote the paper. K.K., T.M. and M.S. contributed to the study design and interpretation of data. Y.U., D.Y. and K.N. contributed to the in vitro shear stress experiments.

\section{Additional information}

Supplementary Information accompanies this paper at http://www.nature.com/ naturecommunications

Competing financial interests: The authors declare no competing financial interests.

Reprints and permission information is available online at http://npg.nature.com/ reprintsandpermissions/

How to cite this article: Banjo, T. et al. Haemodynamically dependent valvulogenesis of zebrafish heart is mediated by flow-dependent expression of miR-21. Nat. Commun. 4:1978 doi: 10.1038/ncomms2978 (2013).

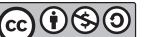

This work is licensed under a Creative Commons AttributionNonCommercial-ShareAlike 3.0 Unported License. To view a copy of this license, visit http://creativecommons.org/licenses/by-nc-sa/3.0/ 